\title{
Toxicomics Report
}

\section{Transcription factor activation in rat primary astrocytes exposed to methylmercury}

\author{
Takuya Takemoto1, Yasuhiro Ishihara1, Mayumi Tsuji², Toshihiro Kawamoto² \\ and Takeshi Yamazaki1 \\ ${ }^{\prime}$ Laboratory of Molecular Brain Science, Graduate School of Integrated Arts and Sciences, Hiroshima University, \\ Higashi-Hiroshima 739-8521, Japan \\ 2Department of Environmental Health, University of Occupational and Environmental Health, Kitakyushu 807-8555, Japan
}

(Received February 17, 2016; Accepted February 25, 2016)

\begin{abstract}
To investigate the adaptive response of astrocytes to an environmental chemical, methylmercury $(\mathrm{MeHg})$, we herein examined transcription factors activated in rat primary astrocytes treated with $10 \mu \mathrm{M} \mathrm{MeHg}$ for $6 \mathrm{hr}$ using the Combo Protein/DNA Array. The activities of 38 transcription factors increased by 5 -fold or greater in response to $\mathrm{MeHg}$. The paired box family of transcription factors were strongly activated by MeHg exposure, which are considered to be activated downstream of NGF and BDNF upregulation induced by MeHg. Nrf-2 (an antioxidant response element) was also activated, which has been reported to act on $\mathrm{MeHg}$ detoxification.
\end{abstract}

Key words: Methylmercury, Astrocytes, Transcription factor network

\section{INTRODUCTION}

Methylmercury $(\mathrm{MeHg})$ is an environmental pollutant that causes neuronal injury and neurological dysfunction. $\mathrm{MeHg}$ is known to accumulate especially in astrocytes in the brain (Aschner et al., 1990), suggesting that astrocytes are a target of $\mathrm{MeHg}$. In fact, gliosis, hyperplasia of activated astrocytes, was observed in the calcarine cortex and cerebellar granular layer of $\mathrm{MeHg}$ intoxicated patients (Shiraki, 1979; Eto et al., 1999). Astrocytes exposed to $\mathrm{MeHg}$ are reported to release interleukin-6 (IL-6) to protect neurons (Noguchi et al., 2013). We recently demonstrated that the treatment of astrocytes with $\mathrm{MeHg}$ induced an upregulation of nerve growth factor (NGF) and brain-derived neurotrophic factor (BDNF), which attenuated MeHg-elicited neurotoxicity (Takemoto et al., 2015). Therefore, $\mathrm{MeHg}$ could activate a network of transcription factors in astrocytes to modulate the gene expression. In this study, we performed a global analysis of the transcription factors which are activated in astrocytes exposed to MeHg using the Combo Protein/DNA Array.

\section{MATERIALS AND METHODS}

\section{Culture of rat primary astrocytes}

All animal procedures were performed in accordance with the Fundamental Guidelines for Proper Conduct of Animal Experiments and Related Activities in Academic Research Institutions under the jurisdiction of the Ministry of Education, Culture, Sports, Science, and Technology (Japan) and the Animal Care and Use Committee of Hiroshima University (Hiroshima, Japan). Pregnant Wistar rats were obtained from Kyudo (Kumamoto, Japan) and were maintained in a temperature-controlled animal facility with 12-hr light-dark cycles.

Cultures of primary astrocytes were prepared from the cerebral cortex of 1 to 2-day-old male Wister rats, as described previously (Ishihara et al., 2015). Briefly, the cerebral cortex was treated with trypsin and DNase I to disperse the cells, which are then seeded on a polyL-lysine coated plate. The purity of the astrocyte-enriched cultures was confirmed by staining with antibodies against the astrocyte-specific marker, glial fibrillary acidic protein (GFAP), and $>97 \%$ of cultured astrocytes showed immunoreactivity to GFAP (data not shown).

\section{Protein/DNA Array}

Rat primary astrocytes were treated with $10 \mu \mathrm{M}$ $\mathrm{MeHg}$ for $6 \mathrm{hr}$, and then cells were collected. Nuclear extracts were prepared as described in our previous report (Ishihara and Shimamoto, 2006). The activity of 345 transcription factors was assessed using the Combo Protein/

Correspondence: Yasuhiro Ishihara (E-mail: ishiyasu@hiroshima-u.ac.jp) 
T. Takemoto et al.

Table 1. Alterations in transcription factor activity in rat primary astrocytes treated with $\mathrm{MeHg}$.

\begin{tabular}{|c|c|c|}
\hline Transcription Factor & Description & Fold change \\
\hline PAX-4 & paired box 4 & 76.4 \\
\hline PAX-6 & paired box 6 & 64.1 \\
\hline ISRE & interferon stimulated response element & 48.7 \\
\hline L-IIIBP & L-III element-binding protein & 38.1 \\
\hline MT-BOX & - & 22.5 \\
\hline PAX-8 & paired box 8 & 19.2 \\
\hline MUSF-1 & mutant upstream stimulatory factor 1 & 17.2 \\
\hline $\mathrm{CBF}$ & core binding factor & 15.8 \\
\hline Freac-2 & forkhead box F2 & 15.7 \\
\hline PAX-3 & paired box 3 & 15.5 \\
\hline LyF-1 & IKAROS family zinc finger 1 & 15.2 \\
\hline HFH-3 & forkhead box I1 & 14.3 \\
\hline CEA & carcinoembryonic antigen & 13.0 \\
\hline $\mathrm{E} 2 \mathrm{~F}-1$ & E2F transcription factor 1 & 12.9 \\
\hline CEBP & CCAAT/enhancer binding protein & 12.5 \\
\hline TFIID & transcription factor II D & 11.9 \\
\hline HOXD-8/9/10 & homeobox D 8/9/10 & 11.8 \\
\hline MEF-1 & myocyte-specific enhancer-binding nuclear factor 1 & 11.3 \\
\hline HFH-2 & hepatocyte nuclear factor $3 / \mathrm{fkh}$ homolog 2 & 11.1 \\
\hline NF-1 & nuclear factor 1 & 10.6 \\
\hline CEF-2 & cardiac enhancer factor 2 & 10.4 \\
\hline GATA-1 & GATA binding protein 1 & 10.4 \\
\hline AP-3 & activator protein 3 & 10.1 \\
\hline ARE & antioxidant response element & 8.8 \\
\hline Pbx1 & pre-B-cell leukemia homeobox 1 & 8.7 \\
\hline GATA-2 & GATA binding protein 2 & 8.6 \\
\hline PARP & poly(ADP-ribose) polymerase & 8.4 \\
\hline c-Rel & v-rel avian reticuloendotheliosis viral oncogene homolog & 8.3 \\
\hline HMG & high mobility group protein & 7.8 \\
\hline E47 & - & 7.3 \\
\hline Afxh/Foxo-4 & forkhead box O4 & 7.3 \\
\hline GFI-1 & growth factor independent 1 & 7.1 \\
\hline EGR-1 & early growth response 1 & 6.7 \\
\hline SMAD-3/4 & SMAD family member $3 / 4$ & 6.6 \\
\hline FAST-1 & forkhead box H1 & 6.3 \\
\hline HNF-1A & hepatocyte nuclear factor 1 homeobox A & 6.2 \\
\hline Ets & - & 6.0 \\
\hline $\mathrm{Sp}-1$ & specificity protein 1 & 5.0 \\
\hline
\end{tabular}

Rat primary astrocytes were treated with $10 \mu \mathrm{M} \mathrm{MeHg}$ for $6 \mathrm{hr}$, and then changes in the activity of transcription factors were evaluated using the Combo Protein/DNA Array. Transcription factors whose activity increased by 5 -fold or greater are listed.

DNA Array (Affymetrix, Santa Clara, CA, USA), according to the manufacturer's instructions. Biotin-labeled DNA-binding probes were incubated with the nuclear extracts. The probes specifically bound to the transcription factors were isolated using spin columns. The eluted, labeled probes were hybridized to a membrane containing an array of 345 transcription factor consensus binding sequences. The array was then washed and incubated with horseradish peroxidase-conjugated streptavidin and subsequently visualized using the ChemiDocTM XRS+ Imaging System (BIO-RAD, Hercules, CA, USA). Data were analyzed using the Image $\mathrm{J}$ software program and the relative activity of transcription factors was calculated.

\section{RESULTS AND DISCUSSION}

Treatment of rat primary astrocytes with $10 \mu \mathrm{M} \mathrm{MeHg}$ for $6 \mathrm{hr}$ did not cause cell death (Takemoto et al., 2015). 
Transactivation of astrocytes by $\mathrm{MeHg}$

The Combo Protein/DNA Array analysis showed that the activity of 38 transcription factors increased by more than 5-fold following exposure to $\mathrm{MeHg}$ compared with untreated cells, as listed in Table 1. Interestingly, the paired box (PAX) family of transcription factors was strongly activated in astrocytes exposed to $\mathrm{MeHg}$ (Table 1). PAX is reported to be activated by NGF and BDNF (Kioussi and Gruss, 1994), and furthermore, we recently revealed that astrocytes treated with $\mathrm{MeHg}$ showed high expression levels of NGF and BDNF (Takemoto et al., 2015). Therefore, PAX activation by MeHg might be an indirect effect of the increased expression of NGF and BDNF elicited by MeHg.

$\mathrm{MeHg}$ is well known to activate nuclear factor erythroid 2-related factor 2 (Nrf-2) by S-mercuration of the cysteine residue of kelch-like ECH-associated protein 1. According to our array analysis, the activation of an antioxidant response element, Nrf-2, was also detected in MeHg-treated astrocytes (Table 1). Nrf-2 is considered to attenuate $\mathrm{MeHg}$-induced neurotoxicity due to an increased expression of anti-oxidative enzymes and/or phase II detoxification enzymes (Kumagai et al., 2013).

As described above, $\mathrm{MeHg}$ increased the expressions of NGF and BDNF in rat primary astrocytes (Takemoto et al., 2015). Activator protein 1 (AP-1) and cAMP response element binding protein (CREB) are well known to upregulate NGF and BDNF, respectively. However, the Combo Protein/DNA Array results indicated that treatment with $\mathrm{MeHg}$ did not affect the transcriptional activity of AP-1 and CREB. In addition, Noguchi et al. (2013) reported that the IL-6 expression also increased downstream of p38 phosphorylation by treatment with $\mathrm{MeHg}$ in astrocytes. Although nuclear factor-kappa B and AP-1 are involved in IL-6 transactivation (Vanden Berghe et al., 1999), the activation of these molecules was not detected in astrocytes exposed to $\mathrm{MeHg}$, indicating that the increased IL-6 expression in the classical pathway is independent of that activation. Collectively, astrocytes could respond to $\mathrm{MeHg}$ via a complex network of transcription factors to protect neurons from harmful stimuli.

\section{ACKNOWLEDGMENTS}

This work was supported in part by a Grant-in-Aid (KAKENHI) to Y.I. and T.Y. (26740024 and 22310041).

Conflict of interest---- The authors declare that there is no conflict of interest.

\section{REFERENCES}

Aschner, M., Eberle, N.B., Goderie, S. and Kimelberg, H.K. (1990): Methylmercury uptake in rat primary astrocyte cultures: the role of the neutral amino acid transport system. Brain Res., 521, 221228.

Eto, K., Takizawa, Y., Akagi, H., Haraguchi, K., Asano, S., Takahata, N. and Tokunaga, H. (1999): Differential diagnosis between organic and inorganic mercury poisoning in human cases--the pathologic point of view. Toxicol. Pathol., 27, 664-671.

Ishihara, Y. and Shimamoto, N. (2006): Involvement of endonuclease $\mathrm{G}$ in nucleosomal DNA fragmentation under sustained endogenous oxidative stress. J. Biol. Chem., 281, 6726-6733.

Ishihara, Y., Takemoto, T., Itoh, K., Ishida, A. and Yamazaki, T. (2015): Dual role of superoxide dismutase 2 induced in activated microglia: oxidative stress tolerance and convergence of inflammatory responses. J. Biol. Chem., 290, 22805-22817.

Kioussi, C. and Gruss, P. (1994): Differential induction of Pax genes by NGF and BDNF in cerebellar primary cultures. J. Cell. Biol., 125, 417-425.

Kumagai, Y., Kanda, H., Shinkai, Y. and Toyama, T. (2013): The role of the Keap1/Nrf2 pathway in the cellular response to methylmercury. Oxid. Med. Cell. Longev., 2013, 848279.

Noguchi, Y., Shinozaki, Y., Fujishita, K., Shibata, K., Imura, Y., Morizawa, Y., Gachet, C. and Koizumi, S. (2013): Astrocytes protect neurons against methylmercury via ATP/P2Y(1) receptor-mediated pathways in astrocytes. PLoS One, 8, e57898.

Shiraki, H. (1979): Neuropathological aspects of organic mercury intoxication, including Minamata disease. Handbook of clinical neurology, 36, 83-145.

Takemoto, T., Ishihara, Y., Ishida, A. and Yamazaki, T. (2015): Neuroprotection elicited by nerve growth factor and brain-derived neurotrophic factor released from astrocytes in response to methylmercury. Environ. Toxicol. Pharmacol., 40, 199-205.

Vanden Berghe, W., De Bosscher, K., Boone, E., Plaisance, S. and Haegeman, G. (1999): The nuclear factor-kappaB engages CBP/ p300 and histone acetyltransferase activity for transcriptional activation of the interleukin- 6 gene promoter. J. Biol. Chem., 274, 32091-32098. 